\title{
TUNABLE MOBILE HANDSET ANTENNA IMPEDANCE MATCH BASED ON RF MEMS BI-STATE CAPACITORS

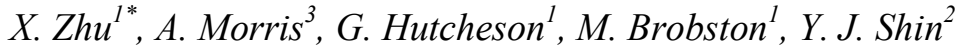 \\ S. Cunningham ${ }^{3}$, J. H. Lee ${ }^{2}$ and J. Hilbert ${ }^{3}$ \\ ${ }^{1}$ Samsung Telecommunications America, Richardson, Texas, USA \\ ${ }^{2}$ Samsung Electronics, Suwon, South Korea \\ ${ }^{3}$ WiSpry, Inc., Irvine, California, USA
}

\begin{abstract}
This paper presents the design, implementation and test result of a Tunable Matching Network (TMN) based on RF MEMS bistate capacitor technology. The targeted application is to improve multi-mode multi-band (MMMB) mobile handset RF performance by providing optimum matching at the antenna RF feed point. The need for such a device is described followed by application requirements. The methodology for selecting topology is discussed, the design flow is illustrated, and the design result is summarized. The RF performance improvement provided by the TMN is demonstrated under both the lab bench, and field test environments.
\end{abstract}

\section{INTRODUCTION}

As mobile communications evolves from $2 \mathrm{G}, 3 \mathrm{G}$ to LTE/LTE-Advanced, and with the booming of the smart phone market, the MMMB RF front end that was previously only available in high-end smart phones has migrated to mid-range even low-end mobile devices. Multi-mode indicates that the handset supports a wide range of communication standards, e.g. GSM, GPRS, EDGE, CDMA, WCDMA, and LTE in latest products. Multi-band denotes that the device is able to operate at a multiple frequency bands, e.g. 700, 800, 900, 1700, 1800, 1900, 2100 and even $2600 \mathrm{MHz}$. The most popular type of antenna in handsets is the PIFA(Planar Inverted F Antenna) type which typically has only 2 resonant frequencies, one at $1900 \mathrm{MHz}$ and the other one at 800 or $900 \mathrm{MHz}$. When a handset operates at any frequency band away from these two resonant bands, the transmit efficiency and receive sensitivity are reduced compared to those at the resonances. Moreover, to obtain maximum radiation/sensitivity to meet stringent carrier RF performance specifications, $\lambda / 4$ structure length is desired leading to a large antenna volume. However, the consumer's want for a slim form factor together with the complexity of hardware due to ever increasing functionality, the large size of display and the large capacity battery has squeezed the available space for the phone antenna. Furthermore, the phone must meet specifications under a range of typical operating conditions, e.g. phone held by hand, phone held by hand and close to head, and phone in flip/slide open position. To make the situation challenging, every phone design has a different physical geometry requirement for the antenna. Therefore, the electrical characteristic of the antenna in each phone is dramatically different. Fig. 1 represents measurement antenna feed point impedance for 5 handsets in different operational conditions for GSM850 band (from 824 to $894 \mathrm{MHz}$ ) which is the lowest frequency band in our design. Some similarity can be observed among these traces. As frequency goes up, the impedance changes more dramatically and the antennas show less resemblance to each other. Usually, a mobile handset antenna is well designed for one operational condition while it will be easily de-tuned under other operational environments, as shown by traces in Fig. 1 swinging from the center to the edge of Smith chart. Meeting all of these requirements within a short product development cycle is difficult and it may not be possible for the engineers to find an acceptable antenna solution. Therefore a general tunable antenna impedance match solution able to be dropped into different product designs to facilitate the product development is preferred.

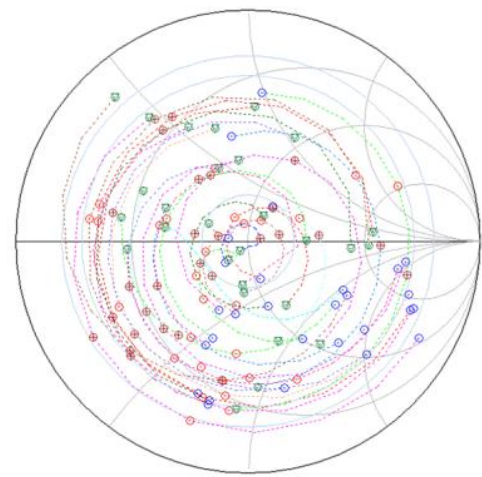

Figure 1: Measured antenna impedance data of 5 different phones under different operation condition at GSM850 band. The blue circles mark the start of TX band, and the red ones label the end of $T X$ band. The red circles with cross mark the start of $R X$ and the green circles with triangle mark the end of $R X$ band.

After decades of development effort, RF MEMS technology finally has matured to provide a viable tunable RF front end solution[1]. Several approaches have explored, e.g. antenna resonant element tuning[2][3] and power amplifier output impedance tuning[4]. However, these solutions are either targeted for a single band or impedance at a single frequency. In this paper, a solution for matching arbitrary impedance simultaneously of transmit and receive is presented.

\section{DESIGN TARGET AND METHODOLOGY}

As aforementioned, the desired TMN device should have the capability to cover the entire Smith chart, but in practice, the impedance within VSWR $\leq 10: 1$ region is specified because it represents majority of measured impedances that need to be matched. One important aspect in TMN design for antenna impedance match is simultaneously matching of TX and RX band impedances. The reason is that CDMA, WCDMA, and LTE FDD all operate using frequency division duplexing(FDD) in which transmit(TX) and receive(RX) signals simultaneously at different frequencies and thus a compromise between of the match for TX and RX has to be made. Whereas for the case of TDD system (such as GSM or LTE TDD) or of PA impedance tuning, only a single frequency at the specified time slot needs to be considered therefore a perfect match can be always achieved theoretically. This simultaneous match of TX and RX impedance makes it impossible to derive a closed-form solution for an arbitrary antenna because there is no general relationship between TX and $\mathrm{RX}$ frequency impedance across antenna designs.

To evaluate matching performance, the term transducer 
gain(TG)[5] or transducer power gain[6], which is widely used in the power amplifier design, is used as the most important criterion to determine the quality of matching,

$$
T G=\frac{\left|S_{21}\right|^{2}\left(1-\left|\Gamma_{S}\right|^{2}\right)\left(1-\left|\Gamma_{L}\right|^{2}\right)}{\left|1-\Gamma_{S} \Gamma_{i n}\right|^{2}\left|1-S_{22} \Gamma_{L}\right|^{2}}
$$

where $\Gamma_{L}$ is the reflection coefficient of the antenna to be matched, $\Gamma_{S}$ is the source reflection coefficient which is zero for perfect $50 \Omega$ source, $\Gamma_{i n}$ is the reflection coefficient of TMN device with the other port connected to the antenna, and $S_{21}$ and $S_{22}$ are TMN Sparameters. The return loss is only used as a monitoring parameter. The rationale is that maximizing the total amount of power radiating out of the antenna, versus minimizing the power reflected by the antenna, determines the performance of the RF front-end; the non ideal reactive component in the network and unwanted resonances can yield a good return loss but inferior transducer gain. To benchmark the antenna performance improvement provided by the TMN device, relative transducer gain is used, which is defined as the ratio between transducer gain with and without the TMN is defined as

$$
R T G=\frac{\left|S_{21}\right|^{2}}{\left|1-S_{22} \Gamma_{L}\right|^{2}}
$$

The selected circuit topology can be explained by a Yin-Yang theory described by Dr. Randy Rhea [7][8] which is shown in Fig 2. A single ' $\mathrm{L}$ ' section of $\mathrm{L}-\mathrm{C}$ network is capable to provide impedances over half of the Smith chart, which implies the conjugate of any impedance in such area can be well matched. Therefore, by cascading Yin, (e.g. Type 1) and Yang (e.g. Type 3), any impedance across the entire Smith chart can theoretically be matched if not considering matching bandwidth (for simultaneous TX and RX impedance match).

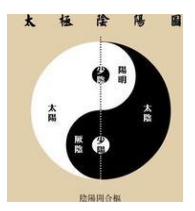

(a)

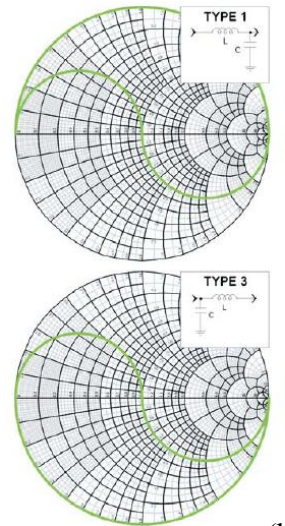

(b)

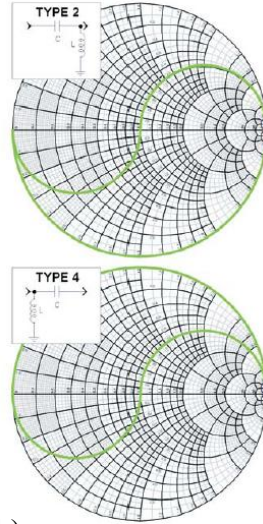

Figure 2: (a) A Chinese Yin-Yang Symbol, (b) The basic concept of Yin-Yang of matching[7].

To implement with available non-ideal reactive components, only 2 sections of L-C are used to minimize the insertion loss. A tunable capacitor is required in parallel with series inductors to achieve a pseudo-tunable inductor. Because any physical capacitor has parasitic inductance and capacitance associated with its RF leads and any tuning element has tuning ratios, the maximum total required capacitance is chosen empirically depending on the coverage and TG requirement. The Type 1 and Type 3 combination is selected following these criteria. A shunt tunable capacitor is added at the common node of two L-C network translating it into the bridged double- $\pi$ circuit topology as shown in Fig. 3(a). One purpose for the added shunt capacitor is to make the network more like a transmission line to mitigate the degradation of impedance match when load impedance falls in VSWR $<2: 1$ circle, especially when operating at high frequency band. The pair of tunable capacitors associated with each inductor is converted into a single capacitor in connecting the two distal series inductor terminals to reduce parasitic at the common node between two inductors and to minimize the total amount of tunable capacitance required. Each capacitor in the schematic is made of a bank of capacitors which is shown in Fig 3(b). Each capacitor in the capacitor bank has only two states, UP state (min. capacitance) and DOWN state (max. capacitance). The Least Significant Bit (LSB) capacitor in the bank determines the tuning resolution of each capacitor bank.

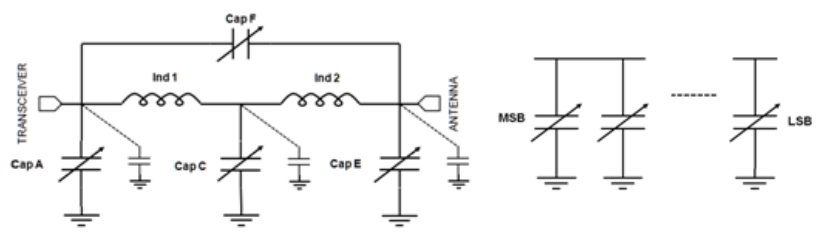

(a)

(b)

Figure 3: (a) Proposed double $\pi$ tunable match network for generic antenna impedance match. (b) Illustration of each tunable capacitor which is made of a bank of capacitors.

An optimization process has been used to determine the value of two inductors and max capacitance for each of five tunable capacitors to maximize impedance coverage over the frequency bands of interest. The minimum capacitance is set by MEMS device inherent tuning ratio. At the initial design stage, the parasitic capacitance was assumed to be a percentage value of the total capacitance at each circuit node and is later replaced with precise parameters derived from HFSS simulation and measurement.

High tuning resolution provides fine tuning steps of impedance. This is primarily required for tuning at high frequency, e.g. $\sim 2 \mathrm{GHz} v s .<1 \mathrm{GHz}$, since tunable component impedance changes more significantly due to the frequency multiplication factor. However, a finer resolution means more physical capacitors are required in the capacitor bank, therefore more parasitic from MEMS capacitors themselves and their interconnections will add to each circuit node which reduces the effective capacitor tuning ratio. A compromise between the best circuitry topology, available resolution and parasitic is made leading to a nominal step size of $1 / 8 \mathrm{pF}$.

\section{SIMULATION RESULTS}

Detailed RF analysis of the RF MEMS capacitor and TMN module substrate is implemented in Ansoft's HFSS, and the entire integrated TMN module simulation is performed in Agilent's ADS. Antenna impedances of multiple handsets under different operation bands and operation conditions have been used to determine the optimum capacitance settings and highest achievable performance. Results are shown in Fig. 4. After the RF MEMS tunable capacitor bank partition and layout are determined, an independent sweep of two inductors within acceptable range is performed to squeeze out more performance, and to balance the high frequency band and low frequency band performance. The result is represented in the contour plot format of RTG as example shown in Fig 5. The green area is preferred region for inductor value selection. 


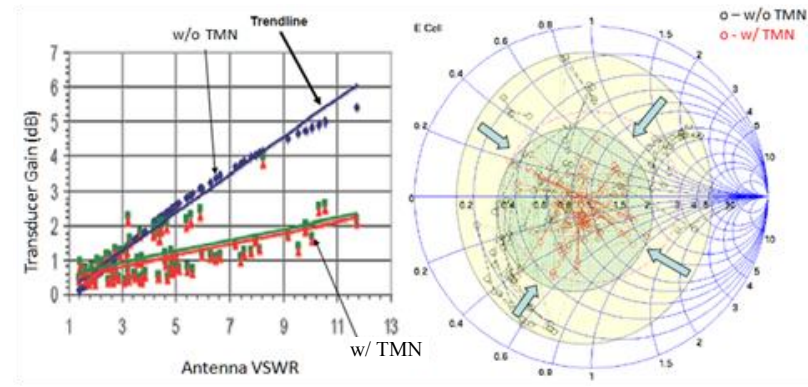

Figure 4: Simulation of antenna performance improvement by $T M N$.

(a) Improvement of TG over wide range of VSWR;

(b) Improvement of $\Gamma$ (dashed line connects $T X$ and $R X$ pairs, green dots connect without TMN and red dots connect with TMN).

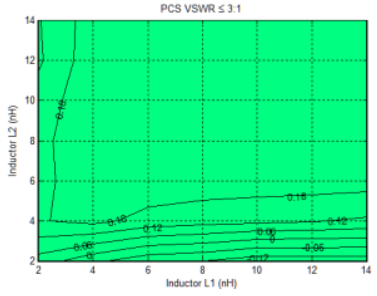

(a)

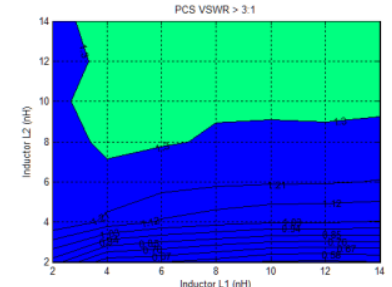

(b)
Figure 5: RTG contour plot by sweeping values of two inductors independently. The green area is preferred region for inductor value selection.

The final simulation result of TMN devices with five different operation band and different operational environments across multiple handset antennas is tabulated in Table 1. The average transducer power gain of $\mathrm{TX}$ and $\mathrm{RX}$ is used to evaluate improvement of the transmit efficiency/receive sensitivity. The data is divided into two regions: a low VSWR region where the antenna is well tuned by design and a high VSWR region where the antenna is de-tuned. In general, the TMN significantly improves the antenna performance in the high VSWR regions while having minimal impact on the RF performance loss in the low VSWR regions. The lower bands show much better improvement than high bands due to two factors: the network is optimized toward low band application which is the greatest challenge for small volume antenna design and the parasitic in the network have less impact at low band than at high band.

Table 1. Simulated average antenna transducer gain improvement with the insertion of TMN RF MEMS device

\begin{tabular}{|l|c|c|}
\hline \multicolumn{1}{|c|}{ Band } & Low VSWR Region & High VSWR Region \\
\hline $800 \mathrm{MHz}$ & -0.09 & 1.33 \\
\hline $900 \mathrm{MHz}$ & 0.11 & 1.63 \\
\hline $1800 \mathrm{MHz}$ & -0.01 & 1.23 \\
\hline $1900 \mathrm{MHz}$ & 0.05 & 0.99 \\
\hline $2100 \mathrm{MHz}$ & -0.23 & 0.78 \\
\hline
\end{tabular}

\section{IMPLEMENTATION}

The high level system design is shown in Fig. 6. As explained above, a bridged double- $\pi$ topology is chosen to provide a general purpose impedance match, although this may not be the best solution for all handset antennas. The motivation is that a stationary hardware platform with programmable control firmware capability will reduce number of handset product development cycles, easing the adoption across other product lines.

Due to limited mobile handset PCB board space and to simplify the control of the MEMS array, a vertically integrated solution RF MEMS above IC[9][10], is chosen. A complete device layout and die photo is shown in Fig. 7. While CMOS control circuitry extends over the entire die, the noisy portion of the digital control and high voltage charge pump is placed to one side of MEMS capacitor banks. This minimizes the electrical interference between CMOS and MEMS portions. The total die size is about $2.2 \times 2.8 \mathrm{~mm}$
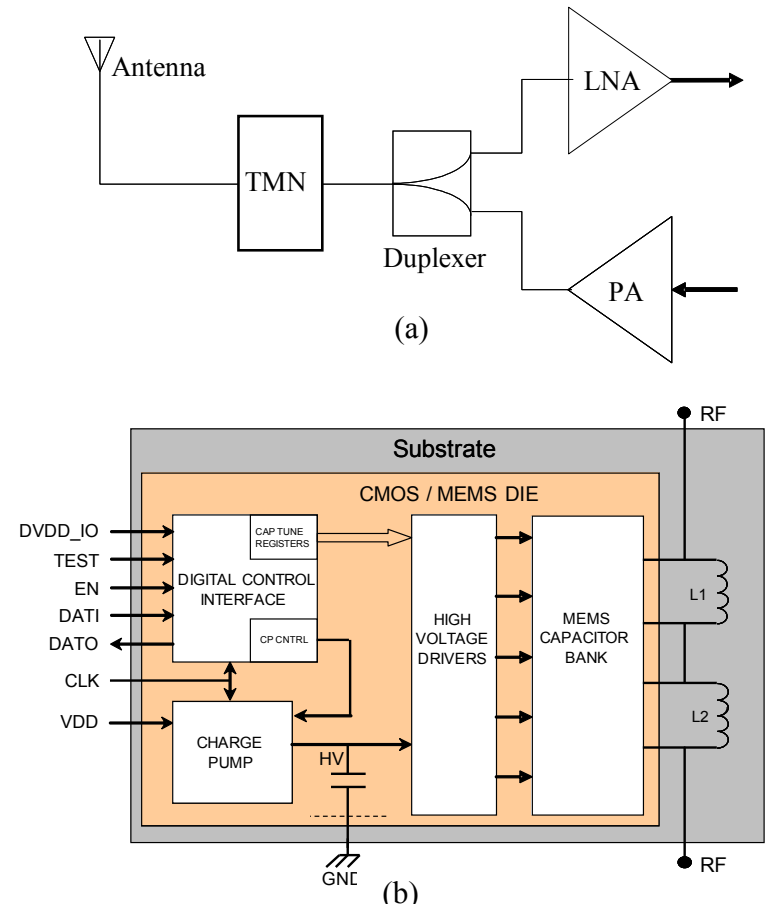

Figure 6: System architecture.

(a) TMN insertion point in the RF front end of mobile device.

(b) High level system schematic of TMN devices.

The bi-state capacitors are fabricated in layers above the CMOS for further isolation from the substrate. The fixed capacitor plate and the fixed actuator plate are fabricated immediately above the CMOS and are covered by a thin, $80 \mathrm{~nm}$, oxide dielectric and ALD $\mathrm{Al}_{2} \mathrm{O}_{3}$. A lower silicon sacrificial layer is deposited and planarized above the fixed capacitor plates. The metal-oxide-metal composite beam is deposited on the lower sacrificial layer. The metal for the fixed capacitor plate and the composite beam is $\mathrm{AlCu}$ with a TiN capping layer. Under the lower beam metal and on top of the silicon sacrificial layer is a $2^{\text {nd }} 80 \mathrm{~nm}$ oxide layer. When the bi-state capacitor is closed, the oxide- $\mathrm{Al}_{2} \mathrm{O}_{3}$-oxide defines the capacitance density, except for a residual air gap. To maintain symmetry of the MEMS beam, an 80nm oxide is deposited on the upper metal. The symmetric metal-oxide-metal composite provides excellent stress and thermal matching to minimize the deformations induced by process stresses or thermal mismatch. 


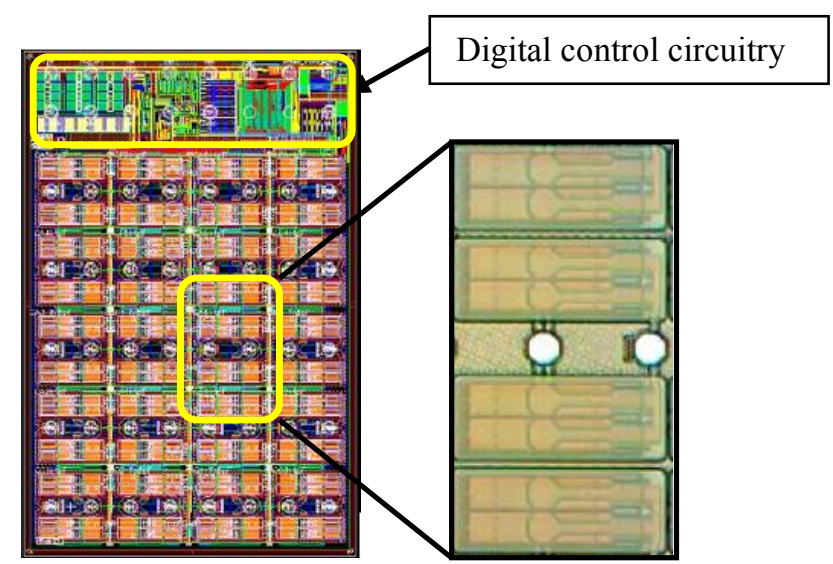

Figure 7: Chip layout and unit cell photo.

Above the MEMS bi-state capacitor, a $2^{\text {nd }}$ silicon sacrificial layer is deposited to form a cavity above the beam. The $2^{\text {nd }}$ silicon sacrificial layer is encapsulated by an oxide layer that is perforated to allow the $1^{\text {st }}$ and $2^{\text {nd }}$ silicon sacrificial layers to be vented by a $\mathrm{XeF}_{2}$ process. Once the silicon is vented, the cavity is sealed by a sub-atmospheric oxide CVD process and passivated with a silicon nitride layer. The wafer level encapsulation process provides a hermetic environment for the operation of the bi-stable capacitor. A cross-section of the MEMS bi-state capacitor is shown in Fig. 8, which shows the capacitor integrated above the HV RF CMOS and shows the hermetic encapsulation above the capacitor.

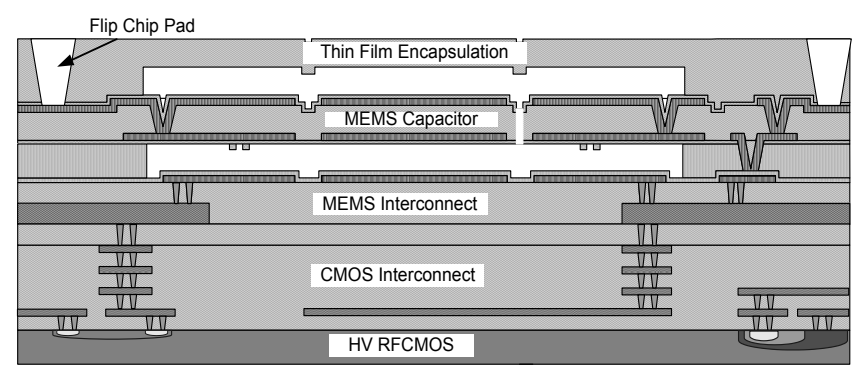

Figure 8: Cross-section of MEMS bi-state capacitor integrated with HV RF CMOS and hermetic encapsulation.

Fig. 7 shows a photograph of a unit cell that is comprised of four bi-state capacitors and two RF flip-chip pads. Each unit cell is defined by its total capacitance, which are comprised of combinations of $0.4 \mathrm{pF}, 0.2 \mathrm{pF}$, and $0.1 \mathrm{pF}$ sized bi-state capacitors. A TMN device is comprised of 20 cells, which are divided among four tunable capacitor banks (Cap A, C, E, and F). The unit cells have 4 capacitor elements that change states simultaneously. The fractional cells are 3-bit binary eight-state cells.

The TMN die includes the integrated CMOS and MEMS bistate capacitors necessary to form the four banks of the bridged double $\pi$ tunable match network. TMN die is flip chip mounted to a laminate substrate, which will include interconnects to connect cells together in the double $\pi$ network configuration. This laminated substrate can be either the phone PCB board or interpose board. The two match network inductors can be designed into the substrate metal layers, which will results in a complete module, or can be designed into the phone PCB with discrete components. The TMN die is shown flip-chip mounted to a laminate substrate in Fig. 9.

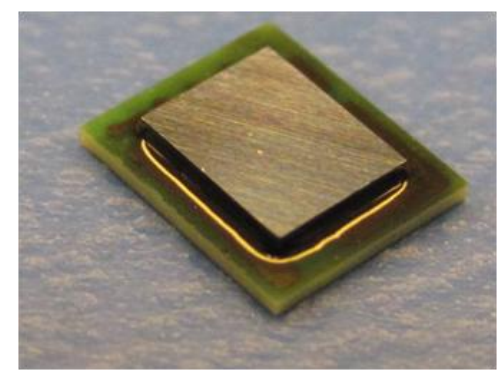

Figure 9: TMN die flip chip mounted to laminate substrate with die underfill.

\section{TEST RESULTS}

The TMN device RF performance is assessed on an evaluation board by two port measurements. The settings of the 5 capacitors on the TMN device is derived by ADS simulation under specified operation frequency band and impedance to be matched. The $50 \Omega$ VNA measured results are converted to specified antenna loading condition to compare with simulation results. Very good correlation between simulation and measurement, both in magnitude and phase, has been demonstrated in Fig. 10.

The device has been integrated into the product devices with one example shown in Fig. 11. This handset has gone through standard TRP/TIS measurement and field tests. Improvement across all band have been observed. For antenna operating at its non-optimized mode, several $\mathrm{dB}$ enhancements has been recorded.
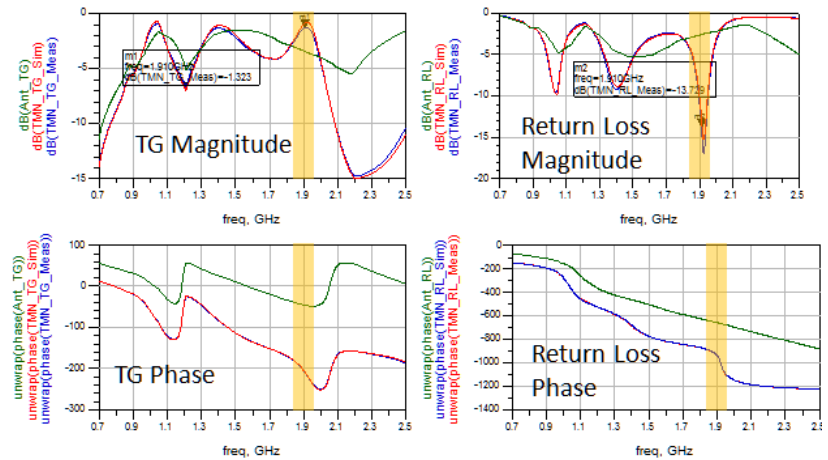

Figure 10: Lab measurement vs. simulation. Green traces are data without TMN device, red traces are simulation data with $T M N$ device and blue traces are measurement data. The vertical yellow stripes mark the operating frequency band where antenna impedance is supposed to be tuned.

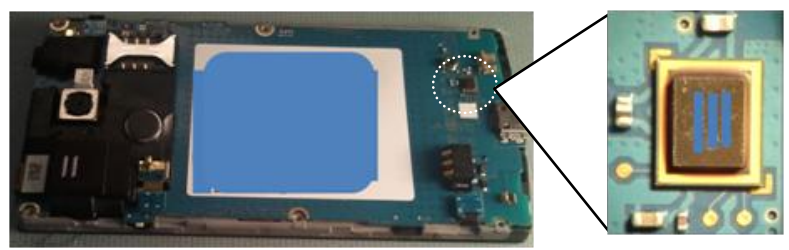

Figure 11: Photos of TMN device and its presence in a mobile handset product.

\section{CONCLUSION}

An RF MEMS tunable capacitor enabled tunable mobile handset antenna impedance match is presented. The device performance can be very well predicted with careful and 
comprehensive design. The results from a general purpose design reveal the capability of RF MEMS tunable devices to improve RF performance for a wide range of antenna product designs. This demonstrates that the RF-MEMS TMN is a viable technology solution to meet MMMB mobile handset challenges. With circuitry topology tailored toward a particular product, even more RF performance improvement is expected but at the cost of longer product development cycle.

\section{ACKNOWLEGEMENT}

We greatly appreciate all of the support and constructive collaboration from various Samsung engineering teams, WiSpry engineering team, Jazz and IBM process development teams.

\section{REFERENCES}

[1] G. M. Rebeiz, "RF MEMS: Theory, Design and Technology," Wiley, New Jersey, 2003.

[2] J. Park, etc., "Tunable planar inverted-F antenna using rf MEMS switch for the reduction of human hand effect," IEEE MEMS Conference, pp. 163-166 (2007).

[3] P. Steeneken, "A Five-Band Reconfigurable PIFA for Mobile Phones," K. Boyle, IEEE Trans. on Antenna and Propagation, Vol. 55, No. 11, pp. 3300-3309, Nov. 2007.

[4] A. van Bezooijen, "Mobile phone performance improvements using an adaptively controlled antenna tuner", 2011 IEEE MTT-S, 5-10 June 2011.

[5] R. Collin, "Foundations of Microwave Engineering," 2nd edition, IEEE press, 2001.

[6] D. Pozar, "Microwave Engineering," 2nd edition, John Wiley and Sons, 1998.

[7] R. Rhea, "The Yin-Yang of Matching: Part 1-Basic Matching Concepts Ying-Yang match", High Frequency Electronics, March 2006.

[8] R. Rhea, "The Yin-Yang of Matching: Part 2-Practical Matching Techniques", High Frequency Electronics, April 2006.

[9] A. K. Stamper, etc., "Planar MEMS RF Capacitor Integration," pp. 1803-1806, Transducers' 11.

[10] S. Natarajan, S. Cunningham, A. Morris, \& D. DeReus, "CMOS Integrated Digital RF MEMS Capacitors", 11th Meeting on Silicon Monolithic Integrated Circuits in RF Systems, Phoenix, AZ, Jan. 17-19, 2011 pp. 173-176.

\section{CONTACT}

*X. Zhu, tel: +1-972-795-9383; xzhu@sta.samsungtelecom.com 